\title{
Durability of First Line Antiretroviral Therapy: Reasons and Predictive Factors for Modifications in a Swaziland Cohort
}

\author{
Simbarashe Takuva ${ }^{1,2,5 *}$, Goedele Louwagie ${ }^{1}$, Khangelani Zuma ${ }^{2,3}$ and Velephi Okello ${ }^{4}$ \\ ${ }^{1}$ University of Pretoria, Faculty of Health Sciences, School of Health Systems and Public Health, Pretoria, South Africa \\ ${ }^{2}$ Mbabane Government Hospital Antiretroviral Therapy Unit, Ministry of Health and Social Welfare, Mbabane, Swaziland \\ ${ }^{3}$ HIVIAIDS, STIs and TB (HAST), Human Sciences Research Council, Pretoria, South Africa \\ ${ }^{4}$ Swaziland National AIDS Program, Ministry of Health and Social Welfare, Mbabane, Swaziland \\ ${ }^{5}$ Clinical HIV Research Unit, Wits Health Consortium, Johannesburg, South Africa
}

\begin{abstract}
Background: Optimizing initial antiretroviral therapy (ART) regimens is critical in improving the durability of treatment efficacy and patient prognosis. Reasons for and risk factors relating to the need for ART modifications were evaluated in an outpatient cohort in Mbabane, Swaziland.

Methods: We examined routine clinical data for 782 patients initiating first-line ART between 1 March 2006 and 31 March 2008. Treatment modification was defined as either a first time single drug substitution or first time regimen switch. Multivariate piecewise Cox regression models were used to identify risk factors for ART modification.

Results: Over a median follow-up period of 21 months, $17.5 \%$ of patients modified their regimen. Drug toxicity (incidence rate of 6.3 per 100 person years $(95 \% \mathrm{Cl} 5.2-7.7)$ ) accounted for $76.6 \%$ of the reasons for modification. Drug contra-indications (incidence rate 9.5 per 100 person years $(95 \% \mathrm{Cl} 6.5-13.9)$ ), namely tuberculosis $(13.1 \%)$ and pregnancy $(6.6 \%)$, accounted for $19.7 \%$ of modifications. In the adjusted multivariate Cox piecewise regression model, beyond 11 months on ART, a baseline CD4 cell count $<200$ cells $/ \mathrm{mm}^{3}$ (HR 4.42; 95\% Cl: 1.62 - 12.1), having stavudine (d4T) in the initial regimen (HR 2.64; 95\% Cl: 1.56 - 4.46), baseline weight > 60kg (HR 2.40; $95 \% \mathrm{Cl}: 1.43$ $-4.04)$ and increase in age (HR 1.03; $95 \% \mathrm{Cl}: 1.00-1.05)$ increased the risk of modification.

Conclusions: Initiating ART earlier, at higher CD4 counts, avoiding drugs with poor safety profiles, such as $\mathrm{d} 4 \mathrm{~T}$, and identifying individuals who may require tuberculosis treatment or may become pregnant could reduce modification rates. This would improve regimen tolerability, while preserving future treatment options.
\end{abstract}

Keywords: First line ART; Regimen durability; Antiretroviral drug modifications; Swaziland

\section{Introduction}

Antiretroviral therapy (ART) use has resulted in a significant reduction of morbidity and mortality associated with AIDS, and scaleup of ART provision in resource limited settings has increased [1]. In Swaziland, a country with one of the highest HIV prevalence rates in the world, ART provision has expanded rapidly, with the result that more than 32,000 patients (out of 58,024 in need of ART) had accessed treatment by the end of $2008[2,3]$.

Studies on North American and European cohorts have shown that most patients tolerate their initial treatment regimens well for up to 24 to 36 months after initiation [4,5]. However, a significant number of patients' treatment regimens are modified for various reasons, including poor drug tolerability, drug toxicities, drug-to-drug interactions, pregnancy and treatment failure [4-7]. In resource limited settings where subsequent treatment options are limited, strategies to maximize drug tolerability and treatment benefits from the available first line regimens are vital. In order to achieve this goal, it is important to understand the reasons for changes in ART regimens and the risk factors that may be associated with a need for treatment modification. Such an understanding will help to individualize drug regimens that are better tolerated among patients while at the same time preserving future treatment options. However, relevant data from resourcelimited settings are still limited. Hence, in this retrospective cohort study, the reasons and risk factors for first line antiretroviral treatment modifications at a large outpatient HIV clinic in Swaziland a country in Southern Africa were investigated.

\section{Methods}

\section{Study setting and participants}

The Mbabane Government Hospital Antiretroviral Therapy Unit is the biggest HIV outpatient clinic in Swaziland. Standard WHO (World Health Organization) - adapted treatment and care guidelines for resource-limited settings are followed [6]. During the study period, 2006 ART guidelines were in use consisting of stavudine (d4T) or zidovudine (AZT) with lamivudine (3TC) and either efavirenz (EFV) or nevirapine (NVP). Patients presenting with WHO Stage 4 disease and/or a CD4 count lower than 200 cells $/ \mathrm{mm}^{3}$ were eligible for the initiation of ART [6]. Clinical and laboratory data were collected at all scheduled visits except for viral load which is only collected when there is suspicion of treatment failure.

For inclusion into this retrospective analysis, patients had to fulfill

*Corresponding author: Dr. Simbarashe Takuva, Epidemiology and Biostatistics Division, Clinical HIV Research Unit, Wits Health Consortium, Private Bag X 2600, Houghton, Johannesburg 2041, South Africa, Tel: +27112768800; Fax: +27114822130; E-mail: stakuva@witshealth.co.za

Received December 08, 2011; Accepted January 24, 2012; Published January 26, 2012

Citation: Takuva S, Louwagie G, Zuma K, Okello V (2012) Durability of First Line Antiretroviral Therapy: Reasons and Predictive Factors for Modifications in a Swaziland Cohort. J Antivir Antiretrovir 4: 014-020. doi:10.4172/jaa.1000040

Copyright: (C) 2012 Takuva S, et al. This is an open-access article distributed unde the terms of the Creative Commons Attribution License, which permits unrestricted use, distribution, and reproduction in any medium, provided the original author and source are credited. 
the following: adults initiated onto first-line ART between 01 March 2006 and 31 March 2008, ART naïve (except for short term Prevention of Mother to Child Transmission of HIV (PMTCT) prophylaxis), at least 2 recorded visits and dates of treatment modification available.

\section{Measurements and data collection}

Three main data sources were used for data collection: an electronic database in Excel format in which patient information is captured at every visit, patient visit files and patients' pharmacy refill booklets. The database contains most demographic and clinical variables for each visit. Most of the patient observations were extracted from this source. Information missing from this source was checked in the patient visit file and pharmacy refill booklet. Data about reasons for ART modifications was mainly extracted from the pharmacy refill booklet and the patient visit file. Information from all the sources was double-entered onto an Epi-Data ${ }^{\mathrm{TM}}$-based questionnaire. This process had quality checks incorporated to reduce data entry errors. Data was then exported to STATA Version $10^{\mathrm{TM}}$ (STATA Corporation, College Station, Texas, USA) for analysis.

The primary outcome was defined as a first-time treatment modification (drug substitution or regimen switch). Time zero was the day of ART initiation and the cut-off date for analysis was 31 December 2008. Patients were right-censored at the date of their last recorded visit if they had stopped clinic visits because of being transferred out, being lost to follow-up or unknown reasons (may have included deaths). The following potential predictor variables were examined: gender, age, weight, CD4 count, WHO stage and ART drug regimen (NVP versus EFV as the NNRTI backbone and d4T versus AZT/TDF as the NRTI backbone) at initiation and time spent on treatment.

\section{Statistical analysis}

Patient demographics and clinical indices at the initiation of treatment were described using percentages for categorical data and medians and inter-quartile ranges for numerical variables. We identified reasons for modification and calculated proportions and incidence rates for these modifications. The log-rank test and KaplanMeier plots were used to compare survival curves between any two compared groups. Hierarchic approach was applied to fit the final model; starting with a null model, followed by fitting the other variables hierarchically alone and then in combination. The model with the best fit (lowest Akaike Information Criteria) was selected. The model was further assessed for the proportionality assumption. A piecewise Cox regression model, whereby we categorized the study observations to two different time periods (during which the hazard ratios of this variable were proportional) was used to deal with the proportionality violation by the $\mathrm{d} 4 \mathrm{~T}$ vs. AZT/TDF variable. The following interaction terms were tested; time period with WHO stage, $\mathrm{d} 4 \mathrm{~T}$ vs. AZT/TDF based regimen, NVP vs. EFV based regimen, and also weight category with $\mathrm{d} 4 \mathrm{~T}$ based regimen vs. AZT/TDF based regimen and CD4 count at initiation. A p-value of less or equal to 0.05 was used to indicate statistical significance. Data was analysed in STATA Version $10^{\mathrm{TM}}$.

This study was approved by the ethics committees of the Swaziland Ministry of Health and Social Welfare and the University of Pretoria, Faculty of Health Sciences.

\section{Results}

\section{Characteristics of study cohort}

Among the 1297 patients who were initiated on first-line ART between 01 March 2006 and 31 March 2008, a total of 824 patients satisfied the inclusion criteria. Of these patients satisfying the inclusion criteria, an additional 28 patients were excluded for missing information on drugs that were modified (or information on whether they were still using their original regimen) and 14 patients were further excluded for missing reasons for ART modification. This left 782 patients for inclusion in the final analysis. The baseline characteristics for the study cohort are summarized in Table 1 . The majority (66.5\%) of these patients were female with a median age of 36 years. The median CD4 count at initiation was 115 (IQR 64-183 cells $/ \mathrm{mm}^{3}$ ), the median weight $62 \mathrm{~kg}$ (IQR $55-70 \mathrm{~kg}$ ), and $25.4 \%$ of the patients were at WHO stage 4 . The most common regimens initiated were NVP/3TC/d4T (36.1\%) and NVP/3TC/AZT (37.9\%). Nearly one fifth of the patients were initiated on EFV/3TC/AZT, whilst $5 \%$ were initiated on EFV/3TC/d4T. Less than $1.6 \%$ of the patients were initiated on a protease-inhibitor based (PI) regimen.

\section{Reasons for treatment modification}

Over a median follow-up period of 21 months (IQR 12.3-27.5), $17.5 \%(n=137)$ of the 782 patients modified their first line antiretroviral regimen. This represented an overall ART modification rate of 0.9 per 100 person years (95\% CI: 0.7-1.0) over a total of 1296 person years of follow-up. Table 2 shows the different reasons for treatment modification. By the study cut-off date, 506 patients (65\%) were still in care. Of the 276 (35\%) patients' no longer in care, 95 (12.1\%) patients were transferred out to other clinic sites and the remaining 181 patients (23.1\%) were either lost to follow up or dead. We had no access to death data.

The incidence rate for modifying treatment for reasons related to drug toxicity was 6.3 per 100 person years (95\% CI: 5.2-7.7). Peripheral neuropathy and lipodystrophy (including lipoatrophy) were the most common toxicity-based reasons, $23.4 \%$ and $22.6 \%$ of all changes

\begin{tabular}{|c|c|}
\hline Characteristics at ART initiation & Summary \\
\hline \multicolumn{2}{|l|}{ Gender } \\
\hline Male, n (\%) & $262(33.5 \%)$ \\
\hline Female, n (\%) & $520(66.5 \%)$ \\
\hline Age (years), median (IQR) & $36(31-43)$ \\
\hline Baseline CD4 count (cells $/ \mathrm{mm}^{3}$ ), median (IQR) & $115(64-183)$ \\
\hline CD4 count $<200$ cells $/ \mathrm{mm}^{3}, \mathrm{n}(\%)$ & $624(82.3 \%)$ \\
\hline CD4 count $\geq 200$ cells $/ \mathrm{mm}^{3}, \mathrm{n}(\%)$ & $134(17.7 \%)$ \\
\hline Baseline weight (kg), median (IQR) & $62(55-70)$ \\
\hline Baseline weight < 60kg, n (\%) & $305(40.8 \%)$ \\
\hline Baseline weight $\geq 60 \mathrm{~kg}, \mathrm{n}(\%)$ & $442(59.2 \%)$ \\
\hline \multicolumn{2}{|l|}{ WHO stage } \\
\hline WHO stage IV, n (\%) & $186(25.4 \%)$ \\
\hline WHO stage I/II/III, n (\%) & $545(74.6 \%)$ \\
\hline \multicolumn{2}{|l|}{ Initial first line ART regimen } \\
\hline NVP-3TC-d4T, n (\%) & $282(36.1 \%)$ \\
\hline NVP-3TC-AZT, n (\%) & $296(37.9 \%)$ \\
\hline EFV-3TC-d4T, n (\%) & $38(4.9 \%)$ \\
\hline EFV-3TC-AZT, n (\%) & $153(19.6 \%)$ \\
\hline Other, $\mathrm{n}(\%)$ & $13(0.7 \%)$ \\
\hline
\end{tabular}

IQR - interquartile range; ART -antiretroviral therapy; NVP - Nevirapine; EFV Efavirenz; 3TC - Lamuvidine; d4T - Stavudine; AZT - Zidovudine; Missing values: Weight $(n=35)$, WHO stage $(n=51)$; CD4 count $(n=24)$; all other covariates $(n=0)$.

Table 1: Baseline characteristics of the 782 patients initiating first-line antiretroviral therapy at the Mbabane Government Hospital in Swaziland between 01 March 2006 and 31 March 2008. 
Citation: Takuva S, Louwagie G, Zuma K, Okello V (2012) Durability of First Line Antiretroviral Therapy: Reasons and Predictive Factors for Modifications in a Swaziland Cohort. J Antivir Antiretrovir 4: 014-020. doi:10.4172/jaa.1000040

\begin{tabular}{|c|c|c|c|}
\hline Reason for modification & Overall & Time period $\leq 11$ months & Time period > 11 months \\
\hline Drug Contraindication, IR (95\% CI) & 9.5 per 100py $(6.5-13.9)$ & 21 per 100py (12.6-34.8) & 5.4 per 100py (3-9.8) \\
\hline TB treatment & 18 & 13 & 5 \\
\hline Pregnancy & 9 & 2 & 7 \\
\hline Toxicity / Side-effects, IR (95\% CI) & 6.3 per 100 py $(5.2-7.7)$ & 22.5 per 100py (16.2-31.4) & 4.6 per 100py (3.7-5.9) \\
\hline Peripheral neuropathy & 32 & 6 & 26 \\
\hline Lipodystrophy / lipoatrophy & 31 & 5 & 26 \\
\hline Anaemia & 10 & 9 & 1 \\
\hline Hypersensitivity rash & 9 & 4 & 5 \\
\hline Hepatitis / raised transaminases & 8 & 2 & 6 \\
\hline CNS disturbances & 5 & 3 & 2 \\
\hline Lactic acidosis & 3 & 0 & 3 \\
\hline Vomiting & 2 & 2 & 0 \\
\hline Gynaecomastia & 2 & 2 & 0 \\
\hline Myalgia & 2 & 1 & 1 \\
\hline Darkening of nails & 1 & 1 & 0 \\
\hline Treatment failure, IR $(95 \% \mathrm{CI})$ & 4.9 per 100py (1.2-19.5) & 10.8 per 100py (1.5-7.7) & 3.2 per 100py (0.4-22.4) \\
\hline Treatment failure & 2 & 1 & 1 \\
\hline Other reasons, IR (95\% CI) & 16.7 per 100py (5.4-52) & 53.5 per 100py (13.3-213.3) & 7.1 per 100py (1-50.2) \\
\hline Drug shortage & 2 & 2 & 0 \\
\hline Pill load & 1 & 0 & 1 \\
\hline Total, IR (95\% CI) & 0.9 per 100py $(0.7-1)$ & 4.8 per 1000py (3.7-6.3) & 0.6 per 100py (4.6-7.0) \\
\hline Total & 137 & 53 & 84 \\
\hline
\end{tabular}

TB-tuberculosis; IR - Incidence rate; 95\% Cl - 95\% Confidence interval; py - person years. Total ( $\mathrm{n}=137)$ : Drug contraindication ( $\mathrm{n}=27,19.7 \%)$; Toxicity ( $\mathrm{n}=105,77 \%$ ); Treatment failure $(n=2,1.5 \%)$ and other reasons $(n=3,2.2 \%)$.

Table 2: Reasons for antiretroviral treatment modification among the 782 patients initiating first-line antiretroviral therapy at the Mbabane Government Hospital in Swaziland between 01 March 2006 and 31 March 2008.

respectively. These were mainly drug substitutions of $\mathrm{d} 4 \mathrm{~T}$ (peripheral neuropathy, $\mathrm{n}=32 \mathrm{~d} 4 \mathrm{~T}$ substitutions, and lipodystrophy, $\mathrm{n}=30 \mathrm{~d} 4 \mathrm{~T}$ substitutions and $\mathrm{n}=1$ AZT substitution). Most patients who had $\mathrm{d} 4 \mathrm{~T}$ substituted because of mitochondrial-related toxicities had the drug replaced by AZT and in some few cases, abacavir (ABC). In cases of peripheral neuropathy, substitution of ART drugs was only after the symptoms did not remit to use of analgesics, amitryptaline and also carbamazepine in some cases. Incidence rate for reasons related to drug contra-indications was 9.5 per 100py (95\% CI: 6.5-13.9) and this was because of TB treatment $(13.1 \%, n=18)$ and pregnancy $(6.6 \%, n=9)$ is contraindicated to use NVP concurrently with rifampicin based TB treatment hence patients on NVP based regimens and also starting TB treatment had NVP replaced with EFV. In pregnant patients, EFV was substituted for NVP in the first trimester because of increased teratogenecity risk. Table 2 further shows these findings stratified by duration on ART.

The majority of the modifications were single-drug substitutions; d4T ( $n=68), \operatorname{NVP}(n=34)$, then AZT $(n=16), \operatorname{EFV~}(n=11)$ and one drug substitution was of ritonivir boosted Saquinavir (SQV/rt) because of a high pill burden. Regimen switches accounted for $5 \%(n=7)$ of the modifications. Of these switches, six were of NVP/3TC/d4T (lactic acidosis, $\mathrm{n}=1$; drug shortage $=2$; treatment failure, $\mathrm{n}=2$; severe rash, $\mathrm{n}=1$ ) and the other one was of NVP/3TC/AZT due to hepatitis.

\section{Risk factors for treatment modification}

Figures 1, 2 and 3 show the Kaplan-Meier estimates for the cumulative probability for modification stratified by initiation regimen (d4T versus AZT/TDF), baseline weight and baseline CD4 count category respectively. The risk for modification over the follow-up period was higher for those on a d4T based regimen, those with higher baseline weight, those initiating ART with a CD4 count $<200$ and among older patients. Gender, WHO stage and being on NVP based regimen did not significantly affect the risk of modification.

As the proportional hazards assumption did not hold, a piecewise Cox's regression model was fitted with two time periods, up to and including 11 months of time on ART and beyond 11 months on ART. This multivariate model was computed to estimate separate hazard ratios before and after 11 months on ART (Table 3). In the first 11 months of therapy, baseline CD $4<200$ versus CD $4 \geq 200$ cells $/ \mathrm{mm}^{3}$ (HR 1.14; 95\% CI: 0.45-2.90), d4T vs. AZT/TDF in the regimen (HR 1.41; $95 \%$ CI: $0.82-2.44$ ), baseline weight $\geq 60 \mathrm{~kg}$ vs. $<60 \mathrm{~kg}$ (HR $1.22 ; 95 \% \mathrm{CI}$ : $0.71-2.11$ ) and female gender (HR 1.26; 95\% CI 0.59-2.70) increased

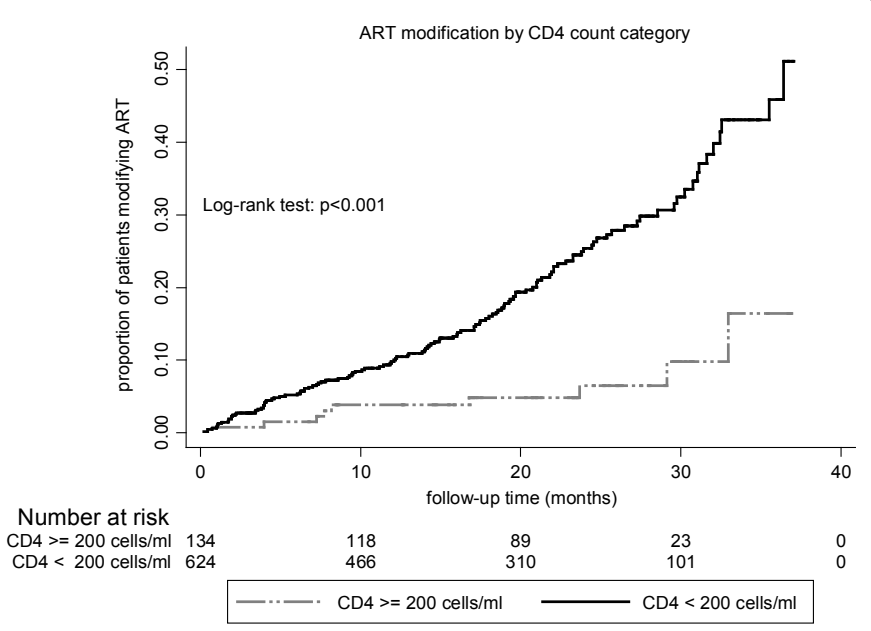

Figure 1: Kaplan-Meier estimates of time to ART modification stratified by CD4 count category at initiation. 
the hazards for treatment modification, but these estimates did not reach statistical significance. A yearly increase in age seemed not to be associated with the risk of treatment modification (HR 0.98; 95\% CI: 0.95-1.02). However, beyond 11 months of ART, the following factors were associated with treatment modification: baseline CD4 $<200$ vs. CD4 $\geq 200$ cells $/ \mathrm{mm}^{3}$ (HR 4.42; 95\% CI: $1.62-12.1$ ), d4T vs. AZT/TDF in the regimen (HR 2.64; 95\% CI: 1.56-4.46), baseline weight $\geq 60 \mathrm{~kg}$ vs. $<60 \mathrm{~kg}$ (HR 2.40; 95\% CI: 1.43-4.04) and increase in age (HR 1.03; 95\% CI: 1.00-1.05). Gender was not significantly associated with the risk of treatment modification.

The following interaction terms were significant in the univariate model; time period with WHO stage, NVP vs. EFV based regimen with time period, and weight category with $\mathrm{d} 4 \mathrm{~T}$ vs. AZT/TDF based regimen. However in the piecewise multivariate models all interaction terms were no longer significant.

\section{Sub-group analysis of d4T-based vs. AZT-based regimens}

We also compared the ART modifications differed according to

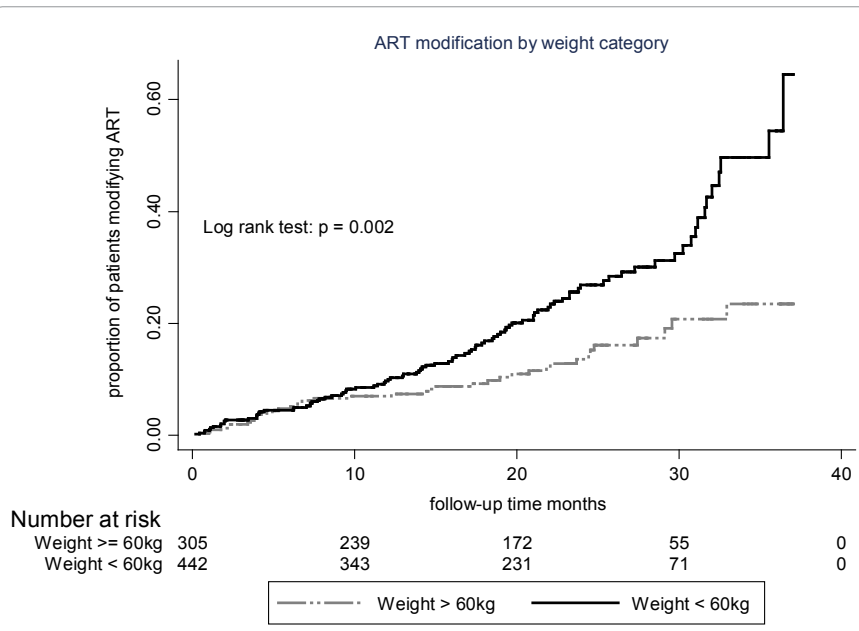

Figure 2: Kaplan-Meier estimates of time to ART modification stratified by weight category at initiation.

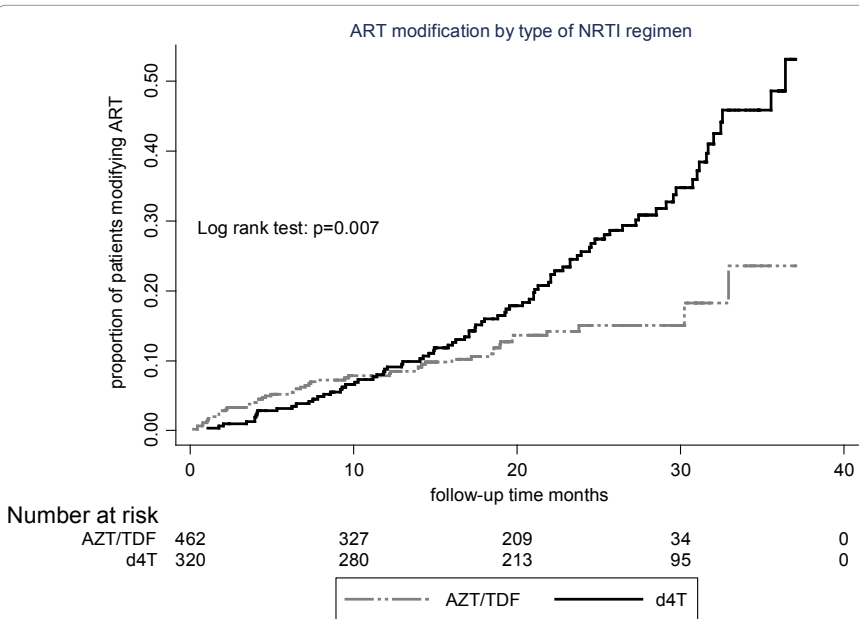

Figure 3: Kaplan-Meier estimates of time to ART modification stratified by type of Nucleoside reverse transcriptase inhibitor in regimen (d4T vs. AZT/TDF in regimen) category at initiation of ART. regimen type. Reasons for modification and hazard ratios for the potential risk factors stratified according to the NRTI in the regimen (on a d4T-based regimen vs. an AZT-based regimen) were computed among patients initiating ART with NVP as the NNRTI. A total of 578 patients (73.9\%) were initiated on NVP-based regimens and 282 out of 578 patients (48.8\%) had d4T in their regimen while 296 out of 578 patients (51.2\%) had AZT in their regimen. Table 4 illustrates these findings.

In the multivariate analysis, baseline CD4 count less than 200cells/ $\mathrm{mm}^{3}$ (HR 5.88; 95\% CI: 1.42-24.3), baseline weight greater then $60 \mathrm{~kg}$ (HR 2.48; 95\% CI: 1.35-4.56) and increase in age (HR 1.03; 95\% CI: 1.01-1.06) were all significant predictors for treatment modification among patients on d4T-based regimens. Among those on AZT-based regimens, only WHO stage (HR 0.38; 95\% CI: 0.62-0.91) showed a significant association with treatment modification.

\section{Discussion}

Over the median follow-up time of 21 months, $17.5 \%$ of the patients in this cohort modified their initial antiretroviral regimen. Reasons for modification were related to toxicity, drug-contraindication, treatment failure, drug shortage and pill load. Beyond 11 months of treatment, having $\mathrm{d} 4 \mathrm{~T}$ in the initial regimen and a baseline weight over $60 \mathrm{~kg}$ increased the risk of modification almost three times, whereas initiating ART with a CD4 count lower than 200 cells $/ \mathrm{mm}^{3}$ increased the risk of treatment modification almost four times. Older age at ART initiation was also associated with increased risk for treatment modification. This study provides unique and important data on reasons for and risk factors involved in antiretroviral treatment modifications in routine clinical set-ups in resource-limited settings where preferred treatment regimens and patient characteristics differ from those widely reported for observational cohorts in Europe and North America and in clinical trial settings.

Only $17.5 \%$ of the patients over the median follow-up period of 21 months modified their treatment. This proportion is far lower than that reported in other observational cohorts, mainly in Europe and North America, where treatment modification rates are higher, 36\% to 53\% [47]. The lack of alternative treatment options in resource-limited settings may influence clinicians to be more reluctant to modify regimens. Also, current guidelines for treatment in resource-limited settings encourage the use of rigid predetermined cost-effective regimens [2,3]. Another issue to consider is that patients in resource-rich settings are more likely to be better informed about their care and would anticipate any unusual effects that could be attributed to their treatment. Such informed patients would be ready to report any intolerance and have their drugs modified.

Toxicity-related factors have been identified as the most common reason for treatment modification in other studies [8-13]. This finding is supported by the results of this study. Gastrointestinal toxicities are the most commonly reported toxicities that result in treatment modification, especially in European and North American cohorts [6$8,10,11,14]$. The frequent use of protease inhibitors (PIs) in developed countries may explain such findings [11]. By contrast, mitochondrialrelated toxicities such as lipodystrophy and peripheral neuropathy were the majority of the toxicities which resulted in treatment modification in the current study. This is due to the widespread use of $\mathrm{d} 4 \mathrm{~T}$ in this clinical set-up, as well as the use of AZT. This is supported by a study in Cape Town, South Africa where the regimens that were used were very similar [9]. In the Cape Town cohort the largest number of drug 
Citation: Takuva S, Louwagie G, Zuma K, Okello V (2012) Durability of First Line Antiretroviral Therapy: Reasons and Predictive Factors for Modifications in a Swaziland Cohort. J Antivir Antiretrovir 4: 014-020. doi:10.4172/jaa.1000040

\begin{tabular}{|c|c|c|c|}
\hline \multirow{2}{*}{ Risk factor } & \multirow{2}{*}{ Crude HR $(95 \% \mathrm{Cl})$} & \multicolumn{2}{|c|}{ Adjusted HR (95\% Cl) } \\
\hline & & Time period $\leq 11$ months & Time period $>11$ months \\
\hline d4T vs. AZT/TDF in regimen & $1.62(1.14-2.30)$ & $1.41(0.82-2.44)$ & $2.64(1.56-4.46)$ \\
\hline NVP vs. EFV in regimen & $1.53(0.98-2.38)$ & - & - \\
\hline CD $4<200$ vs. CD $4 \geq 200$ cells $/ \mathrm{mm}^{3}$ & $3.38(1.72-6.65)$ & $1.14(0.45-2.90)$ & $4.42(1.62-12.1)$ \\
\hline Weight $\geq 60$ vs. $<60 \mathrm{~kg}$ & $1.82(1.26-2.65)$ & $1.22(0.71-2.11)$ & $2.40(1.43-4.04)$ \\
\hline Female vs. male & $1.34(0.93-1.95)$ & $1.26(0.59-2.70)$ & $1.56(0.86-2.85)$ \\
\hline WHO stage I/II/III vs. IV & $0.96(0.56-1.58)$ & - & - \\
\hline Age (per year) & $1.01(1.26-2.66)$ & $0.98(0.95-1.02)$ & $1.03(1.00-1.05)$ \\
\hline d4T vs. AZT/TDF in regimen ${ }^{*}$ Time period & $0.98(0.97-1.00)$ & - & - \\
\hline d4T vs. AZT/TDF in regimen*Weight category & $2.61(1.85-3.66)$ & - & - \\
\hline NVP vs. EFV in regimen ${ }^{*}$ Time period & $0.96(0.95-0.99)$ & - & - \\
\hline WHO stage I/II/III vs. IV*Time period & $0.96(0.95-0.98)$ & - & - \\
\hline CD $4<200$ vs. CD $4 \geq 200$ cells $/ \mathrm{mm}^{3 *}$ Time period & $1.00(0.93-1.02)$ & - & - \\
\hline
\end{tabular}

HR - Hazards ratio; 95\% Cl - 95\% Confidence interval; NVP - Nevirapine; EFV - Effavirenz; AZT - Zidovudine; d4T - Stavudine; TDF - Tenofovir; Weight category weight $\geq 60$ vs. $<60 \mathrm{~kg}$.

Table 3: Risk factors for ART modification among the 782 patients initiating first-line antiretroviral therapy at the Mbabane Government Hospital in Swaziland between 01 March 2006 and 31 March 2008.

\begin{tabular}{|c|c|c|c|}
\hline Reason for modification & Overall & $\mathrm{d} 4 \mathrm{~T}$ in regimen & $\mathrm{AZT}$ in regimen \\
\hline Drug Contraindication, IR $(95 \% \mathrm{CI})$ & 10.3 per 100py (6.9-15.4) & 10 per 100py (5.6-18.1) & 10.6 per 100py (6.1-18.2) \\
\hline TB treatment & 18 & 6 & 12 \\
\hline Pregnancy & 6 & 5 & 1 \\
\hline Toxicity / Side-effects, IR (95\% CI) & 5.9 per 100py (4.8-7.3) & 5.1 per 100 py (4-6.5) & 11 per 100py (7.2-16.7) \\
\hline Peripheral neuropathy & 29 & 29 & 0 \\
\hline Lipodystrophy / lipoatrophy & 27 & 26 & 1 \\
\hline Anaemia & 6 & 0 & 6 \\
\hline Hypersensitivity rash & 9 & 2 & 7 \\
\hline Hepatitis / raised transaminases & 8 & 3 & 5 \\
\hline Lactic acidosis & 3 & 3 & 0 \\
\hline Vomiting & 2 & 0 & 2 \\
\hline Myalgia & 1 & 0 & 1 \\
\hline Treatment failure, IR $(95 \% \mathrm{CI})$ & 4.9 per 100py (1.2-19.5) & 4.9 per 100py (1.2-19.5) & - \\
\hline Treatment failure & 2 & 2 & 0 \\
\hline Other reasons, IR (95\% CI) & 53.3 per 100py (13.3-213.3) & 53.3 per 100py (13.3-213.3) & - \\
\hline Drug shortage & 2 & 2 & 0 \\
\hline Total, IR (95\% Cl) & 1 per 100py (0.8-1.2) & 1.2 per 100py $(0.9-1.5)$ & 0.7 per 100py $(0.5-1)$ \\
\hline Total & 113 & 78 & 35 \\
\hline
\end{tabular}

TB-tuberculosis; IR - Incidence rate; $95 \% \mathrm{Cl}-95 \%$ Confidence interval; py - person years; $d 4 \mathrm{~T}$ - Stavudine; AZT- Zidovudine. Totals for Drug contraindication ( $\mathrm{n}=24$ $21.2 \%)$; Toxicity $(n=85,75.2 \%)$; Treatment failure $(n=2,1.8 \%)$; Other reasons $(n=2,1.8 \%)$

Table 4: Reasons for antiretroviral treatment modification among the 578 on Nevirapine based regimens patients initiating first-line antiretroviral therapy at the Mbabane Government Hospital in Swaziland between 01 March 2006 and 31 March 2008.

substitutions due to toxicity was in patients on $\mathrm{d} 4 \mathrm{~T}$ : approximately $21 \%$ of those who originally started with a d4T-based regimen substituted it, because of toxicity. The findings of the study reported in this article raise questions about the continued role of $\mathrm{d} 4 \mathrm{~T}$ in first-line treatment guidelines and support recommendations to move away from such regimens [12]. Recently, Zambia a country with a similar clinical and demographic setting to Swaziland, has introduced tenofovir (TDF) as an essential part of its first-line regimens. Preliminary safety and tolerability results are encouraging [13].

Treatment modification due to anaemia related to AZT toxicity were significant ( $7.3 \%$ of the modifications). This highlights the need to screen patients for anaemia before the initiation of therapy. In the DART trial, in which patients with low haemoglobin were excluded $(\mathrm{Hb}<8 \mathrm{~g} / \mathrm{dl})$, the majority of patients who had incident anaemia did not have baseline anaemia even though lower haemoglobin did correlate as a risk factor. Only $6.6 \%$ of patients developed anaemia with only
$11 \%$ having to switch [15]. In our cohort, patients had only initiated AZT containing regimens if there haemoglobin at baseline was $>10 \mathrm{~g} /$ dl. NVP in a treatment regimen was well tolerated, accounting for less than $10 \%$ of the reasons for modification. This figure approximates the findings in a study by Boulle et al. [10] which showed that $8.8 \%$ of patients initiating NVP in their cohort changed to EFV. An analysis of the Khayelitsha cohort in South Africa also showed a similar trend [16]. These findings contrast with those in rich-resourced settings, where NVP use is discouraged, especially in patients with higher CD4 counts $[17,18]$. However, patients in the Mbabane ART clinic and in similar settings usually start on ART with lower CD4 counts. Considering the widespread NVP use in this setting and changes in CD4 criteria for ART initiation, more studies are required to determine the safety and tolerability of NVP-containing regimens in such settings.

In contrast with the findings of other reported studies, gender was not a significant risk factor for treatment modification in this 
population $[9,19,20]$. Older age in this cohort showed association with treatment modification, a finding which was not seen in other related studies $[9,10]$. This association with age was however weak. Weight upon initiating ART was a very strong risk factor for modification. This may be a reflection of the practice of weight-based $\mathrm{d} 4 \mathrm{~T}$ dosing during the study accrual period. It is important to note that during this period for patients with a baseline weight over $60 \mathrm{~kg}$ it was recommended to initiate a higher dose of $\mathrm{d} 4 \mathrm{~T}$ ( $40 \mathrm{mg}$ if their weight was over $60 \mathrm{~kg}$ versus $30 \mathrm{mg}$ if their weight was less than $60 \mathrm{~kg}$ ) [2]. Doses of $\mathrm{d} 4 \mathrm{~T}$ above $30 \mathrm{mg}$ are no longer recommended, as they have been shown to have a worse toxicity profile, besides achieving a similar efficacy to the $30 \mathrm{mg}$ dose [21]. Heavier patients have also been shown by some studies to be more susceptible to mitochondrial-related toxicities $[18,21]$.

Where other treatment options are not available, a risk score for toxicity and tolerability could be useful in pre-determining treatment regimens. Patients initiating ART with lower CD4 counts also showed poor regimen durability, compared to those with higher CD4 counts at initiation. A baseline CD4 count $<200$ cells $/ \mathrm{mm}^{3}$ increased the risk treatment modification by more than four times, compared to a baseline $<200$ cells $/ \mathrm{mm}^{3}$. These results are consistent with other findings that show that sicker patients are more likely to have more side-effects and more regimen changes, possibly because they are on other medications for opportunistic infections, than those with a higher CD4 count at initiation [22,23]. Poor regimen durability shown in this sub-group of patients initiating ART also provides evidence that initiating ART earlier, at CD4 counts well above 200 cells $/ \mathrm{mm}^{3}$ should be the current standard of care. Besides improving patients' clinical prognosis, this approach will also improve the ART regimen durability.

Drug contra-indications mainly due to drug-drug interactions with TB treatment and the diagnosis of pregnancy during ART also contributed to modification in this study. Patients initiating TB therapy; and a diagnosis of pregnancy during therapy constituted $13 \%$ and $6.6 \%$ of the reasons for modification respectively. This demonstrates the burden of $\mathrm{TB}$ in this setting and raises the need for more aggressive TB screening in patients initiating ART. Family planning programs should also be routinely integrated into routine HIV care to prevent unplanned and unwanted pregnancies. Patients most likely to become pregnant need to be identified early on during their treatment. These findings confirm recent findings by a recent study in Côte d'Ivoire [24] in which the rate of treatment modifications was 20.7 per 100 patient- years ( $\mathrm{n}=2012$ patients). The most frequent drug substitutions were due to intolerance (12.4 per 100 patient-years), pregnancy (4.5 per 100 patient-years) and TB (2.5 per 100 patient-years). Twenty percent of EFV substitutions resulted from pregnancy and $18 \%$ of NVP substitutions were related to TB treatment.

Our findings must be interpreted in light of their limitations. A retrospective review of medical records carries a risk of misclassification bias. The analysis of a single treatment site, even though it is the largest in the country, may only reflect treatment practices at that centre, which cannot be generalized to other sites. Another limitation is the possibility of informative censoring bias. Events resulting in censorship could have been related to the outcome of the study, i.e. patients lost to follow-up might have been lost due to poor drug tolerability or even treatment failure. This limitation implies that the modification rates may have been underestimated. This limitation may also partially explain the very low treatment failure rate we observed. It is highly likely that there were other treatment failures not documented due to patients lost to follow-up or dying (about 23.1\%). Since there was no active programme for follow up tracing, we had no access to detailed follow up data to examine in order to understand the magnitude of this possible bias. The other important limitation of this study is that it reflects prescribing practices relative to a 2-year period (2006-2008), which is no longer common practice. However these results still reinforce some already known data about poor $\mathrm{d} 4 \mathrm{~T}$ tolerability and the increased risk of toxicity and regimen tolerability for individuals initiating ART with low CD4 counts [25,26].

In conclusion, this study reports lower rates of ART modifications in this population than in cohorts in resource-rich settings. However, the burden of peripheral neuropathy and lipodystrophy related to $\mathrm{d} 4 \mathrm{~T}$ use is a matter of major concern, as it accounts for the majority of modifications. Another concern is that a significant number of patient's treatment was modified due to TB therapy and pregnancy. Safer and more tolerable drugs (e.g the introduction of TDF in Swaziland treatment guidelines in 2010) should be made more accessible to treatment programs in resource-limited settings. Screening for TB should be intensified and routine before patients start HAART. Family planning programs should also be integrated into routine HIV care. Addressing these issues will further reduce modifications and improve initial ART regimen tolerability, thereby increasing the probability of achieving sustained viral response, and at the same time preserving future treatment options.

\section{Authors' Contributions}

ST and GL conceived the study, participated in its design, statistical analysis and helped to draft the manuscript. KZ participated in the design of the study and the statistical analysis. VO participated in the design and coordination of the study. All authors read and approved the final manuscript.

\section{Acknowledgements}

We would like to thank the patients and personnel of the Mbabane Government Hospital Antiretroviral Therapy Unit who made this project possible. In particular we would like to express many thanks to Dr Marianne Calnan for her useful comments on the initiation of the study. We are also thankful to the Swaziland government and the Global Fund to Fight Tuberculosis, HIV and Malaria who have made the widespread provision of antiretroviral therapy to HIV-infected patients in Swaziland a reality.

\section{References}

1. Swaziland Demographic Health Survey Report 2008 (2008) Ministry of Health and Social Welfare/Measure DHS Project.

2. Antiretroviral therapy for HIV infection in adults and adolescents (2006) Recommendations for a public health approach. World Health Organization.

3. Ministry of Health and Social Welfare, Swaziland (2006) National Guidelines for Antiretroviral Treatment and Post-exposure Prophylaxis for Adults and Adolescents.

4. Van Roon EN, Verzijl JM, Juttmann JR, Juttmann JR, Lenderink AW, et al (1999) Incidence of discontinuation of highly active antiretroviral therapy (HAART) and its determinants. J Acquir Immune Defic Syndr Hum Retrovirol 20: 290-294.

5. Fellay J, Boubaker K, Ledergerber B, Bernasconi E, Furrer H, et al. (2001) Prevalence of adverse events associated with potent antiretroviral treatment: Swiss HIV Cohort Study. Lancet 358: 1322-1327.

6. Hammer SM, Squires KE, Hughes MD, Grimes JM, Demeter LM, et al. (1997) A controlled trial of two nucleoside analogues plus indinavir in persons with human immunodeficiency virus infection and CD4 cell counts of 200 per cubic millimeters or less. N Engl J Med 337: 725-733.

7. d'Arminio Monforte A, Lepri AC, Rezza G, Pezzotti P, Antinori A, et al. (2000) Insights into the reasons for discontinuation of the first highly active antiretrovira (HAART) regimen in a cohort of antiretroviral naive patients. I.CO.N.A. Study Group. Italian Cohort of Antiretroviral-Naïve Patients. AIDS 14: 499-507. 
Citation: Takuva S, Louwagie G, Zuma K, Okello V (2012) Durability of First Line Antiretroviral Therapy: Reasons and Predictive Factors for Modifications in a Swaziland Cohort. J Antivir Antiretrovir 4: 014-020. doi:10.4172/jaa.1000040

8. Kirstein LM, Greenblatt RM, Anastos K, Levine A, French AL, et al. (2002) Prevalence and correlates of highly active antiretroviral therapy switching in the Women's Interagency HIV Study. J Acquir Immune Defic Syndr 29: 495-503.

9. Boulle A, Orrel C, Kaplan R, Van Cutsem G, McNally M, et al. (2007) Substitutions due to antiretroviral toxicity or contraindication in the first 3 years of antiretroviral therapy in a large South African cohort. Antivir Ther 12: 753760 .

10. Australian HIV Observational Database (2002) Rates of combination antiretroviral treatment change in Australia ,1997-2000. HIV Med 3: 28-36.

11. Deeks SG, Smith M, Holodniy M, Kahn JO (1997) HIV-1 protease inhibitors. A review for clinicians. JAMA 277: 145-153.

12. Rapid advice: antiretroviral therapy for HIV infection in adults and adolescents (2009) World Health Organization.

13. Chi BH, Mwango A, Giganti M, Mulenga LB, Tambatamba-Chapula B, et al. (2003) Early clinical and programmatic outcomes with tenofovir-based antiretroviral therapy in Zambia. J Acquir Immune Defic Syndr 54: 63-70.

14. Kirk O, Gerstoft J, Pedersen C, Nielsen H, Obel N, et al. (2001) Low body weight and type of protease inhibitor predict discontinuation and treatment limiting adverse drug reactions among HIV infected patients starting a protease inhibitor regimen: consistent results from a randomized trial and an observational cohort. HIV Med 2: 43-51.

15. Ssali F, Stöhr W, Munderi P, Reid A, Walker AS, et al. (2006) Prevalence, Incidence, predictors and timing of severe anaemia following initiation of zidovudine containing regimens in adults with HIV infection within the DART Trial. Antivir Ther 11: 741-749.

16. Coetzee D, Hildebrand K, Boulle A, Maartens G, Louis F, et al. (2004) Outcomes after two years of providing antiretroviral treatment in Khayelitsha, South Africa. AIDS 18: 887-895.

17. Centers for Disease control and prevention (2001) Serious adverse events attributed to nevirapine regimens for post exposure prophylaxis after HIV exposures - worldwide, 1997-2000. MMWRMorb Mortal Wkly Rep 49: 11531156 .

18. Wit FW, Weverling GJ, Weel J, Jurriaans S, Lange JM (2002) Incidence and risk factors for severe hepatotoxicity associated with antiretroviral therapy. J Infect Dis 186: 23-31.

19. Youle M, Sawyer W (1998) For the HIV Health Economics Collaboration Reasons for discontinuation of antiretroviral treatment: A clinical survey. AIDS 12: 186

20. Dieleman JP, Jambroes M, Gyssens IC, Sturkenboom MC, Stricker BH, et al. (2002) Determinants of recurrent toxicity-driven switches of highly active antiretroviral therapy. The ATHENA Cohort. AIDS 16: 737-745.

21. Hill A, Ruxrungtham K, Hanvanich M, Katlama C, Wolf E, et al. (2007) Systematic review of clinical trials evaluating low doses of stavudine as part of antiretroviral treatment. Expert Opin Pharmacother 8: 679-688.

22. The Strategies for Management of Antiretroviral Therapy (SMART) Study Group, El-Sadr WM, Lundgren JD, Neaton JD, Gordin F, et al. (2006) CD4+ count-guided interruption of antiretroviral treatment. N Engl J Med 355: 22832296.

23. Kitahata MM, Gange SJ, Abraham AG, Merriman B, Saag MS, et al. (2009) Effect of early versus deferred antiretroviral therapy for HIV on survival. N Engl J Med 360: 1815-1826.

24. Messou E, Anglaret X, Duvignac J, Konan-N'dri E, Komena E, et al. (2010) Antiretroviral treatment changes in adults from Cote d'Ivoire: the roles of tuberculosis and pregnancy. AIDS 24: 93-99.

25. HänselA, Bucher HC, Nüesch R, Battegay M (2001) Reasons for discontinuation of first highly active antiretroviral therapy in a cohort of proteinase inhibitornaïve HIV-infected patients. J Acquir Immune Defic Syndr 26: 191-193.

26. Mocroft A, Youle M, Moore A, Sabin CA, Madge S, et al. (2001) Reasons for modification and discontinuation of antiretrovirals: results from a single treatment centre. AIDS 15: 185-194.
Submit your next manuscript and get advantages of OMICS Group submissions

Unique features:

- User friendly/feasible website-translation of your paper to 50 world's leading languages

- Audio Version of published paper

Digital articles to share and explore

Special features:

200 Open Access Journals

15,000 editorial team

21 days rapid review process

Quality and quick editorial, review and publication processing

Indexing at PubMed (partial), Scopus, DOAJ, EBSCO, Index Copernicus and Google Scholar etc

Sharing Option: Social Networking Enabled

- Authors, Reviewers and Editors rewarded with online Scientific Credits

Better discount for your subsequent articles

Submit your manuscript at: http://www.editorialmanager.com/virology 